\title{
Prävention und Glaubwürdigkeit von Gesundheitspolitikern: ein Minenfeld
}

\author{
Die Ablehnung der Initiative «Schutz vor Passivrauchen» durch den Nationalrat im \\ Dezember 2011 und durch den Ständerat im März 2012 bestätigt unverständlicher- \\ weise nachträglich ein mangelhaftes Bundesgesetz.
}

Rainer M. Kaelin

\begin{abstract}
* Die Literaturhinweise finden sich in der Online-Ausgabe unter www.saez.ch $\rightarrow$ Aktuelle Nummer oder $\rightarrow$ Archiv $\rightarrow 2012$ $\rightarrow 23$.
\end{abstract}

Korrespondenz:

Dr. med. Rainer M. Kaelin

2, place Hôtel-de-Ville

CH-1110 Morges

rmkaelin[at]sunrise.ch
Gesundheitsprävention ist ein explosives Thema. Dass die Tabakprävention seit Jahren massiv durch die Tabaklobby beeinflusst wird, ist kaum anzuzweifeln. Dass aber die Bundesparlamentarier sich so von ihrem Auftrag ablenken lassen, dass sie die Initiative «Schutz vor Passivrauchen» ablehnen, stimmt nachdenklich: die Abstimmungsresultate sind ein verspäteter Applaus an das Bundesgesetz vom Oktober 2008.

\section{Der Werdegang des Bundesgesetzes zum Schutz vor Passivrauchen von 2008}

2004 reichte der Parlamentarier und Medizinprofessor Felix Gutzwiller (FDP/ZH) die Initiative «Schutz der Bevölkerung und der Wirtschaft vor dem Passivrauchen» mit dem Ziel eines rauchfreien Arbeitsplatzes ein. Im selben Jahr unterzeichnete Bundesrat Pascal Couchepin die Rahmenkonvention der WHO zur Eindämmung des Tabakgebrauches [1] ${ }^{*}$, die festhält: «Die Vertragsparteien erkennen an, dass wissenschaftliche Untersuchungen eindeutig beweisen, dass Passivrauchen Tod, Krankheit und Invalidität verursacht.» - Eine Subkommission der Gesundheitskommission des Nationalrates (SGK-N) erarbeitete einen Gesetzesentwurf zum Schutz vor Passivrauchen, der im Juni 2007 vom Präsidenten der SGK-N, Pierre Triponez (FDP/BE), vorgelegt wurde: «Mit dem vorgeschlagenen Bundesgesetz zum Schutz vor Passivrauchen wird ein Paradigmawechsel herbeigeführt. Arbeitsplätze und geschlossene Räume, die der Öffentlichkeit zugänglich sind, sollen neu grundsätzlich rauchfrei sein. Diese Regelung betrifft auch Gastronomiebetriebe» [2]. Die damalige SGK-N stimmte mit 16 zu 3 Stimmen für ein Spezialgesetz (und nicht für eine Änderung des Arbeitsgesetzes).

Etappenweise wurde das hehre Projekt demontiert: Am 4.10.2007, der Minderheit der SGK-N folgend, nahm der Nationalrat eine Vorlage an, nach der sich öffentliche Lokale als Raucherlokale deklarieren dürfen, falls eine klare Trennung zwischen Raucher- und Nichtraucherzonen nicht möglich oder nicht zumutbar ist [3]. Obwohl sich die ständerätliche Gesundheitskommission «grundsätzlich zustimmend zum Schutz vor Passivrauchen positionierte» [4], suchte sie danach, das Arbeiten in solchen Raucherräumen zuzulassen. So wurde im Stän- derat (Bruno Frick, CVP/SZ) eingefügt, dass Raucherräume und Fumoirs bedient sein dürfen, falls im Arbeitsvertrag das Personal ausdrücklich zustimmt. Das heutige Bundesgesetz bewahrt nur das Prinzip des rauchfreien öffentlichen Raums, jedoch mit zahlreichen Ausnahmen: Falls der Gastraum kleiner ist als $80 \mathrm{~m}^{2}$, darf der Wirt entscheiden, ob sein Lokal Raucherlokal sein soll. Raucherräume sind zugelassen, sie sind grundsätzlich nicht bedient, ausgenommen die Angestellten stimmen schriftlich ihrer Verräucherung zu. Das Gesetz gilt in der ganzen Eidgenossenschaft, ausser in Kantonen mit strengeren Regeln.

Das Gesetz wurde im Nationalrat mit $89 \mathrm{zu}$ 88 Stimmen und im Ständerat durch Stichentscheid seines Präsidenten Christoffel Brändli, damaliger Präsident von santésuisse, äusserst knapp verabschiedet. Es ist strenggenommen gar kein Bundesgesetz, sondern ein Minimal(Alibi)gesetz (siehe Kasten nächste Seite), da es ausdrücklich vorsieht, dass die Kantone weitergehende Regelungen erlassen können.

\section{Debatte und Entscheid des Nationalrates vom 22. 12. 2011}

Angesichts des dergestalt seiner Substanz beraubten Gesetzes war es logisch, dass eine Allianz von Gesundheits- und Ärzteorganisationen, darunter die FMH (www.rauchfrei-ja.ch, [6]) die Volksinitiative «Schutz vor Passivrauchen» lancierte, die im Mai 2010 eingereicht wurde. Die Ablehnung der Initiative durch den Nationalrat mit 118 gegen 55 Stimmen (und durch den Ständerat, der mit 26 gegen 15 Stimmen ablehnte, sie an die Kommission zurückzuweisen) ist eine nachträgliche klare Unterstützung des Bundesgesetzes von 2008. Dies ist umso erstaunlicher, als das Gesetz damals nur sehr knapp angenommen worden war. Wie lauten die Argumente, die anscheinend heute so viel besser überzeugen als vor 4 Jahren?

- Frau Humbel Naef (CVP/AG) spricht für die SGK-N, welche die Initiative mit 14 gegen 8 Stimmen ablehnt: Sie findet «problematisch», das Gesetz wieder zu ändern, obwohl festgehalten wird, dass 15 Kantone weitergehende Regeln kannten, schon bevor das Bundesgesetz verabschiedet worden war. Der Initiative wird dennoch vorge- 
worfen, «unverhältnismässig» und «verfrüht» zu sein.

- Die Initianten werden verunglimpft, «der Zwängerei» und des Verstosses «gegen Treu und Glauben» bezichtigt (Daniel Fässler, CVP/AI; Sylvia Flückiger-Bäni SVP/AG). Diese Volksvertreterin, Vorstandsmitglied des Gewerbeverbandes, erinnert an die «grosse soziale Rolle» des Gasthauses, was für sie Grund ist, dass sich Angestellte verräuchern lassen sollten.

- Die Argumentation nimmt pathetische Züge an: «Ich bin für ein Rauchverbot im öffentlichen Raum. Aber: Weil die Gesundheit für die allermeisten von uns ein sehr wichtiges Gut ist, müssen die Entscheide von jedem Einzelnen getroffen werden. In einer Gemeinschaft, die ihre Mitglieder als unmündig (sic!) erachtet, kann und soll der Gesetzgeber die Verantwortung für die zu treffenden Beschlüsse nicht übernehmen» (Roland Büchel, SVP, SG). Verworren die Argumentation von Rusconi Pierre (SVP/TI): «Das Tessin ist mit dem guten Beispiel vorangegangen (...) Das hat uns auch die Chance einer schönen Eintracht zwischen Rauchern und Nichtrauchern gebracht (...) Wenn wir aber die Perfektion erreichen wollen, indem wir weiterfahren, alles und jedes $\mathrm{zu}$ reglementieren (...), dann wird unser Perfektionismus unsere Freiheit zerstören. (...) Vielleicht ist es noch nicht der gute Zeitpunkt, einen weiteren Schritt vorwärts zu tun. Der bisher vorgegebene Beweis ist ganz und gar genügend. Dennoch lade ich Sie ein, diese Initiative noch nicht - ich lege den Akzent auf noch nicht anzunehmen.»

\section{«Wir rufen dem Gesetzgeber in Erinnerung, was normal}

ist: Jedermann, auch in unserem Land, hat das Recht, eine saubere, rauchfreie Luft zu atmen.»

- Ein Verstoss gegen Verfassung und WHO-Rahmenkonvention wird suggeriert: «Es geht darum, die Kohärenz in unserer Rechtsordnung zu bewahren. Die heute geltende Gesetzgebung wurde im Geiste des Postulates der WHO-Rahmenkonvention erlassen und unter Beachtung des Proportionalitätsprinzips (in) unserer Bundesverfassung. Als Nichtraucher habe ich die Tyrannie der Raucher erlitten, ... Muss man deswegen eine Tyrannie durch eine andere ersetzen?» (Mauro Poggia (GE/ parteilos).

\section{Ein unausgewogener Wettstreit}

Gegen solche Sophisterei waren die 19 an der Debatte teilnehmenden Nationalrätinnen und -räte, die sich für die Initiative aussprachen, machtlos.

\section{Kritik am Bundesgesetz von 2008} während seiner Erarbeitung [5]

- Bundesrat Pascal Couchepin verteidigte die Lösung, wie sie von der Volksinitiative «Schutz vor Passivrauchen» verfolgt wird: «Die wirtschaftlichen Argumente sind, ehrlich gesagt, nicht seriös! Niemand (kann) bestreiten, dass Passivrauch etwas Negatives ist. Ich lade Sie ein, für eine Lösung zu stimmen, welche nicht freiheitsbeschränkend ist, die ganz einfach gesund ist, und sich auf den Vorschlag des Bundesrates zu einigen.»

- Konrad Graber (CVP/LU): «Wozu sollen wir ein Bundesgesetz erlassen, welches dazu führt, dass die Kantone aktiv werden? Wenn keine gesamtschweizerische Lösung erreicht wird, kann man sich wirklich fragen, wozu man überhaupt ein Gesetz erlassen soll.»

- Rolf Büttiker (FDP, SO): «Stellen Sie sich einmal vor, in diesem Land 26 unterschiedliche Lösungen zu propagieren und zu installieren! (...) Das wäre staatspolitisch bedenklich. Deshalb werde ich auch als Liberaler konsequent der harten Linie zustimmen, so, wie sie der Bundesrat vorgezeichnet hat.»

- Urs Schwaller (CVP/FR): (...) dass sich die Begeisterung für das neue Gesetz in der ständerätlichen Kommission in Grenzen gehalten hat (...) Das Resultat von 4 zu 3 Stimmen bei 3 Enthaltungen zeigt, dass wir auch schon überzeugter und (...) bessere Gesetzgebungsarbeit geleistet haben.»

- Felix Gutzwiller (FDP/ZH): «... der Arbeitnehmerschutz (muss) unteilbar sein. Es gibt keinen Schadstoff, bei dem man zwei Arten von Arbeitsplätzen schaffen würde, bei dem man sagen würde: Bitte, liebe Arbeitnehmerinnen und Arbeitnehmer, Sie sind frei, einen Arbeitsplatz mit oder einen Arbeitsplatz ohne Schadstoff zu belegen.» «Die Branche hat am Anfang eine arbeitsrechtliche Regelung bekämpft, immer mit dem Hauptargument, man wolle keine Ungleichheiten. Es gibt sehr viele Abgrenzungsprobleme. Es gibt Gastronomiebetriebe, die Lebensmittel herstellen, es gibt Gastronomiebetriebe in Einkaufszentren, die an sich rauchfrei sind, dort (darf man) dann nicht rauchen, währenddem man nebenan rauchen darf. Das ist doch keine solide Gesetzgebung.»

Ihre Voten, neben den bekannten wissenschaftlichen Gesundheitsargumenten [7] waren einleuchtend: «Der Inhalt der Initiative kann nicht so unvernünftig sein, denn er ist identisch mit dem Vorschlag der SGK von 2007; der Kommission, die die Gesundheit im Fokus hat. Wenn es nicht das Ziel jedes Unternehmens ist, seine Mitarbeiterinnen und Mitarbeiter vor Passivrauchen zu schützen (...), sol- 


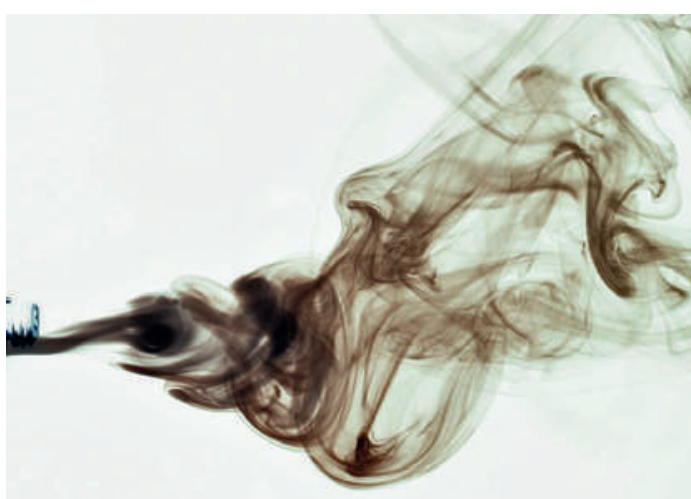

Die Professionellen aller Gesundheitsberufe sind aufgerufen, im Herbst zum Abstimmungserfolg der Volksinitiative «Schutz vor Passivrauchen» beizutragen.

che Gesundheitsrisiken sind sicher zuerst zu beheben» (Maja Ingold, EVP/ZH). Und: «Dieser Umgang mit dem Gesundheitsschutz ist eine Farce. (...) Es ist doch nicht fair, dass wir unter dem Feigenblatt der Freiwilligkeit die Verantwortlichkeit für den Gesundheitsschutz am Arbeitsplatz auf die Schultern der Arbeitnehmerinnen und Arbeitnehmer abwälzen; dies ist eine rückwärtsgerichtete Entwicklung. Herr Bundesrat, ich wundere mich darüber, dass sich ein Gesundheitsminister gegen die Verbesserung des Gesundheitsschutzes am Arbeitsplatz einsetzt; können Sie ausschliessen, dass eine Arbeitskraft aus dem Gastgewerbe Sanktionierungen in Form von Einstelltagen der Arbeitslosenversicherung erhält, wenn sie von ihrem Recht Gebrauch macht und die Arbeit in einem Raucherlokal ablehnt, und deshalb ihre Stelle verliert?» (Max Chopart-Acklin, SP/AG).

\section{Didier Burkhalter:}

\section{die unverständliche Stellungnahme}

Eine brauchbare Antwort ist der im Dezember 2011 amtierende Gesundheitsminister dem Nationalrat und der Schweizer Bevölkerung schuldig geblieben. Im Vergleich zu seinem Amtsvorgänger und Parteikollegen Couchepin, der sich für ein solches Gesetz schämen würde [8], ist seine Stellungnahme diplomatisch [9]: Er gibt zu, dass die geltende Regelung unvollständig ist, dass sie hinter der damals vom Bundesrat vertretenen Lösung zurückbleibt, dass der Rückgang der akuten Koronarsyndrome auf die vorher erlassenen strengeren kantonalen Regelungen zurückzuführen sind, (und nicht auf das schon bei seiner Einführung überholte Bundesgesetz). Und empfiehlt die Ablehnung der Initiative!

\section{Lobbying und Interessenkonflikte}

- Die im Parlament ausgebreiteten Argumente rechtfertigen die gewaltig gewachsene Unterstützung des Bundesgesetzes im Vergleich mit seiner Annahme 2008 nicht. Dass Lobbying die entscheidende Rolle spielt [10], erscheint begründet: Die heutige Situation kommt ausschliesslich der Zigarettenindustrie zugute. Ihre Botschaft «Passivrauch ist gar nicht so schlimm» wird durch ein bundesstaatlich abgesegnetes Gesetz verstärkt. Kantonale Regelungen können mit dem Hinweis auf das Bundesgesetz aufgeweicht werden, und Umgehungsversuche wie Raucherklubs gewinnen dadurch juristische Kaution. Die Banalisierung des Rauchens gegenüber Jugendlichen $[11,12,13]$ durch die Tabakindustrie wird mit einem eidgenössischen Text offizialisiert und ist erfolgreich, wie die neuesten Zahlen [14] erkennen lassen: Trotz Abnahme der Gesamtraucherzahlen auf $27 \%$, stagniert der Rückgang seit 2008; und seit dem Jahre 2010 rauchen mehr Jugendliche von 14 und 15 Jahren als zuvor.

- Die Abstimmung [15] zeigt, dass im Parteienspektrum die SVP, die Bürgerlichen Demokraten und die Grünliberalen am diszipliniertesten (ohne Gegenstimme und Enthaltung) gegen eine wirksame Passivrauchgesetzgebung gestimmt haben, gefolgt von den Liberal-Radikalen und den Christdemokraten, während die Sozialdemokraten und die Grünen die Initiative unterstützt haben. Es wurde schon darauf hingewiesen [16, 17], dass Interessenverflechtungen eine Rolle spielen, wie die «Allianz der Wirtschaft für eine Massvolle Präventionspolitik» (www.awmp.ch, [18]) zeigt. Im Jahre 2008 vom Schweizerischen Gewerbeverband gegründet, zählt sie zu ihren Mitgliedern die seit Jahren durch die Tabakindustrie infiltrierte Gastrosuisse [19], den Verband von Zigarrenfabrikanten und des Tabakwarenhandels, unterstützt von SVP, CVP und «Swiss Cigarette», hinter der sich die drei in der Schweiz etablierten Tabakmultinationalen verbergen.

- Voten der Nationalratsdebatte vom 22.12.2011 zeigen, dass diese Kreise Druck ausüben: «Die ausserparlamentarische Kommission für Tabakfragen (hat) aufgerufen, die Lücken beim Passivrauchen endgültig zu schliessen. Diese mehr als problematische Einmischung (...) wirft gravierende staatspolitische Fragen auf (...) Kommt dazu, dass solche Kommissionen völlig einseitig zusammengesetzt sind (...) Während Personen, die den Tabakkreisen zugerechnet werden, in der Kommission nicht vertreten sind, gehören mehrere Mitglieder der Kommission der Lungenliga an ...» (Flückiger-Bäni, SVP/AG). Und: «Wie kommt es, dass (die Eidgenössische Tabakkommission), die vom Bund bezahlt und vom Bund beaufsichtigt wird, nach der bundesrätlichen Stellungnahme ihre Ansichten und Meinungen vertritt und sich klar gegen den Bundesrat stellt?» (Roland Borer, SVP/SO).

Die systematische Demontage des von der Gesundheitskommission 2007 vorgeschlagenen Paradigmenwechsels und die Ablehnung der Initiative 
«Schutz vor Passivrauchen» durch Bundesrat und Parlament schaden der Glaubwürdigkeit der Bundesorgane. Sie sollten die Gesundheitsinteressen der Bevölkerung vor die der Tabakindustrie stellen können. Dies ist nicht eine Frage der Parteipolitik, sondern der intellektuellen Redlichkeit. Wir sind beunruhigt, dass sich Parlamentarier instrumentalisieren lassen, eine Sachverständigenkommission gerade deswegen zu kritisieren, weil sie sich unbehindert durch Inter- essenkonflikte äussert. Wir rufen dem Gesetzgeber in Erinnerung, was normal ist: Jedermann, auch in unserem Land, hat das Recht, eine saubere, rauchfreie Luft zu atmen. Da die Volksvertreter Mühe haben, diese Offensichtlichkeit zu erkennen, wird dies demnächst das Volk tun. Die Professionellen aller Gesundheitsberufe sind aufgerufen, zum Abstimmungserfolg der Volksinitiative beizutragen!

\section{Dr Erik Allgöwer, FMH Médecine interne, Yverdon-les-Bains}

Dr. med. Jürg Barandun, Gründer und Leitender Partner Lungenzentrum Hirslanden Zürich

PD Dr. Jürg Barben, Präsident Lungenliga St. Gallen. Leitender Arzt Pneumologie/Allergologie. Ostschweizer Kinderspital, St. Gallen

Prof. Chris T. Bolliger, Professor of Medicine, Director Respiratory Research, Co-chairman Division of Pneumology, Faculty of Health Sciences, University of Stellenbosch, Cape Town, South Africa

Dr. med. Antonio Bonfiglio, FMH Allgemeinmedizin, Vorstandsmitglied SGAM, Langnau a.A.

Dr. med. Otto Brändli, Lungenarzt, Präsident Swiss Lung Fundation

Dr. med. Johannes Brühweiler, FMH Innere Medizin, Vorstandsmitglied Hausärzte Schweiz, Zürich

Prof. Dr. med. Martin Brutsche, Chefarzt Pneumologie und Zentrum Schlafmedizin, St. Gallen

Prof. em, Jean-Claude Chevrolet, ancien médecin chef des soins intensifs des HUG, Genève

Dr. med. Bernard Egger, Pneumologie FMH, médecin chef, Hôpital de Rolle/VD

Dr Nicolas Eisenhoffer, Pédiatrie FMH, Genolier

Dr. med. Margot Enz Kuhn, FMH Allgemeinmedizin, Vorstandsmitglied SGAM, Baden

Dr. med. Ingo Fengels, Pneumologie FMH, Vizepräsident und Ligaarzt, Lungenliga Luzern-Zug, Vorstandsmitglied Krebsliga Zentralschweiz

Prof. Dr Jean-William Fitting, Médecin chef, Service de Pneumologie, CHUV, Lausanne

Dr Jean-Georges Frey, Pneumologie FMH, Médecin Sous-directeur, Centre valaisan de Pneumologie Montana

PD Dr. med. Christophe von Garnier, Leitender Arzt, Universitäts-Kinderklinik für Pneumologie, Inselspital Bern

Dr Stephen Garronne, FMH Pneumologie et Médecine interne, Monthey/VS

Prof. em Dr. Peter Gehr, Institut für Anatomie, Leiter des Nationalen Forschungsprogrammes 64

(Chancen und Risiken von Nanomaterialien) SNF, Bern

Dr Sandor Györik, Viceprimario Medicina interna, Ospedale Regionale Bellinzona e Valli, Bellinzona

Dr Gaudenz Hafen, médecin associé, MER, responsable de l'unité de pneumologie pédiatrique CHUV, Lausanne

Prof. Dr. med. Jürg Hammer, Leitender Arzt, Chefarzt - Stellvertreter Pädiatrie, Intensivmedizin und Pneumologie, Universitäts-Kinderspital beider Basel

Dr. med. Andrea L. Hartmann, Facharzt für Arbeitsmedizin, Winterthur

Dr. med. Erich Helfenstein, Pneumologie und Innere Medizin FMH, Luzern

Dr. med. Markus Hofmann, Allgemeinmedizin, Arbeitsmedizin FMH, Frauenfeld

Dr François Héritier, Président de la Société Suisse de Médecine Générale, Vice-président des Médecins de famille Suisse, Courfaivre JU

Dr. med. Peter Kälin, Allgemeine Medizin FMH, Sportmedizin SGSM, Manualmedizin SAMM,

Präsident der Ärztinnen und Ärzte für Umweltschutz, Leukerbad

Dr. med. Rainer M. Kaelin, Pneumologie und Innere Medizin FMH, Morges, Mitglied des Initiativkomitees

Dr Philippe Kehrer, Pneumologie FMH, Genève

Dr. med. Bruno Knöpfli, FMH Pädiatrie, Pneumologie und Sportmedizin, Davos 
Prof. Dr. med. PhD. Nino Künzli, Vizedirektor Schweizerisches Tropen- und Public Health Institut Universität Basel, Mitglied Initiativkomitee

Dr. med. Max Kuhn, Leitender Arzt Pneumologie Kantonsspital Graubünden, Chur

Dr. med. Rolf Lindt, Lyss

Dr. med. Stefan Minder, Pneumologie FMH, Salemspital, Bern

PD Dr. med. Alexander Möller, Leiter Pneumologie, Universitäts-Kinderkliniken Zürich

Dr. med. Marc Müller, FMH Innere Medizin,

Vorstandsmitglied Schweizerische Gesellschaft für Allgemeinmedizin und Hausärzte Schweiz, Grindelwald

Prof. Dr Laurent Nicod, Médecin Chef Division de Pneumologie, CHUV, Lausanne

Dr Reto Olgiati, Médecine interne et Pneumologie FMH, Délémont JU

Dr. med. Helmut Oswald, FMH Kinder- und Jugendmedizin, spez. Lungenkrankheiten, Winterthur

Prof. Dr Arnaud Perrier, Médecin chef de service, Médecine interne Générale, Hôpital Universitaire, Genève

Dr. med. Max Pfenninger, Pneumologie FMH, Olten

Dr Franco Quadri, Medicina interna e malattie polmonari FMH. Capo Servizio di Pneumologie,

Ospedale Regionale Bellinzona e Valli, Bellinzona

Dr. med. Markus Riederer, FMH Pneumologie und Innere Medizin, Leitender Arzt Medizin RSE, Burgdorf, Präsident des Vereins der Pneumologen des Kantons Bern

Dr Philippe Rieder, FMH Médecine interne et Pneumologie, Médecin chef Hôpital de St-Loup, VD

Prof. Dr Thierry Rochat, Médecin Chef, Service de Pneumologie, HUG, Genève

Dr Jean-Pierre Roulin, FMH Pneumologie, Fribourg

Dr. med. Jürg Rufener, FMH Innere Medizin, Vorstandsmitglied SGIM/SSMI, Interlaken

Prof. Dr. med. Ralph Alexander Schmid, Universitätsklinik für Thoraxchirurgie, Inselspital Bern

Prof. Dr. med. Martin Schöni, Chefarzt Ambulante Pädiatrie, Universitäts-Kinderklinik, Inselspital Bern

Dr Ariane Studer, Médecine interne générale FMH, Lausanne

Prof. Dr. Michael Tamm, Chefarzt Pneumologie, Universitätsspital, Basel

Dr Bernard Thiévent, Pneumologie FMH, Porrentruy

Prof. Dr. med. Robert Thurnheer, Pneumologie FMH, Scherzingen

Dr Christophe Uldry, Médecin Chef, Service de Pneumologie et Réhabilitation Hôpital de Rolle

Dr. med. Silvia Ulrich Somaini, PD, FMH Pneumologie, Zürich

Dr. med. Beat Villiger, Pneumologie FMH, Ärztlicher Leiter Medizinisches Zentrum Bad Ragaz

Dr Jacques Wacker, Médecine interne et Pneumologie FMH, La Chaux-de-Fonds

Dr Renato Waldenburger, Chefarzt Innere Medizin und Pneumologie FMH, Kantonales Spital Appenzell Ausserrhoden

Dr Jean Pierre Zellweger, FMH Pneumologie, Fribourg 


\section{Literatur}

1 www. who.int/tobacco/framework

2 Parlamentarische Initiative «Schutz der Bevölkerung und der Wirtschaft vor dem Passivrauchen.» Bericht der Kommission für soziale Sicherheit und Gesundheit des Nationalrates. Vom 1. Juni 2007.

3 M.Gugger, Th. Cerny, J. Barben, W. Karrer, R.M.Kaelin: Glaubwürdigkeit!, SAeZ 2008;89: 1/2. 22-23.

4 Medienmitteilung der ständerätlichen Kommission für soziale Sicherheit und Gesundheit vom 9.01.2009 über die Sitzung vom 8./9.2008.

5 Die Voten der Politiker sind aus den Wortprotokollen der Parlamentsdebatten zitiert, www.admin.ch/ Nationalrat.

6 Die Allianz besteht aus 53 Gesundheitsorganisationen Ärztegesellschaften, die FMH, die Lungenliga Schweiz, die Krebsliga Schweiz usw. siehe www.rauchfrei-ja.ch/ de/allianz-komitee/allianzpartner.html

7 Bonetti PO, Trachsel LD, Kuhn MU, Schulzki T, Erne P, Radovanovic D, Reinhart H. Incidence of actuel myocardial infarction after implementation of a public smoking ban in Graubünden, Switzerland. Two year follow-up. Swiss Med Wkly, 2011;141:w13206. www.smw.ch

8 Pascal Couchepin, am 14.9.2008, Ständerat: «si au moins législation il y a, qu'elle soit simple et qu'elle ne provoque pas des disputes supplémentaires à propos du contrat de travail avec les collaborateurs et à propos des surfaces. La solution de la minorité est plus simple. Je m'excuse de vous avoir indigné, Monsieur Schwaller. J'en suis désolé, j'en ai honte, mais finalement, j'aurais honte de la législation, si elle passait telle que la majorité la propose.»

9 Didier Burkhalter, am 22.12.2011, Nationalrat: «... le handicap le plus sérieux, c'est le manque de protection d'une partie des employés de la restauration avec le service dans les fumoirs. (...) Mais comme on l'a vu, la loi actuelle permet de protéger une très grande partie de la population, tant sur les lieux de travail que dans les lieux fermées accessibles au public. L'initiative irait donc plus loin, de manière nationale.» Und: «Le conseil fédéral s'est battu en 2007 et 2008 pour une législation qui allait même plus loin que celle votée finalement par le parlement. Mais le parlement a décidé (...) (et), la mise en application a été faite il y a peu de temps. Elle se passe bien et les effets sont positifs.»

10 Barben J, Borer H, Brändli O, Kaelin RM und zahlreiche Mitunterzeichner. Glaubwürdigkeit II: Das Minenfeld der Prävention. Schweiz Ärztezeitung. 2008;89(38):1614--6.

11 Im Internet finden sich Agenturen, welche für Jugendliche private Parties sponsern, was Speis und Trank, sowie Zigaretten beinhaltet.

12 Die Tabakindustrie lädt junge Erwachsene ein, um «ihre Produkte zu testen».

13 Systematisch wird durch die Tabakindustrie Fernsehund Filmsponsoring betrieben. Mehrere in Thorax, Vol 66. 10, Oct 2011 erschienene Artikel dokumentieren die Wirksamkeit dieser Anstrengungen: Waylen AE et al. Cross-sectional association between smoking depictions in films and adolescent tobacco use in a British cohort study; Hunt K et al. Exposure to smoking in films and own smoking among Scottish adolescents: a cross-sectional study. Morgenstern $\mathrm{M}$ et al. Smoking in movies and adolescent smoking: cross-cultural study in six European countries.

14 Keller R, Radtke T, Krebs H, Hornung R. Der Tabakkonsum der Schweizer Wohnbevölkerung in den Jahren 2001-2010. Zusammenfassung des Forschungsberichtes 2011. Psychologisches Institut Universität Zürich.

15 Gemäss elektronischer Stimmabgabe stimmten für die Ablehnung der Volksinitiative (Nationalrat, 22.12.2011):

Grün-Lib.: 9 ja/0 nein; Enth.: 0

Chr.-Dem.: 19 ja/6 nein; Enth.: 2

Bürg.Dem.: 8 ja/0 nein; Enth.: 0

Grüne 3: ja/10 nein; Enth.: 2

Freisinn: 25 ja/0 nein; Enth.: 1

SP: 2 ja/39 nein; Enth.: 4

SVP 51ja/0 nein; Enth.: 0

Total: 118 ja/55 nein; Enth.: 9

16 Kaelin RM, Diethelm P. «Voting of members of Swiss Parliament during elaboration of federal smoking ban of Oct. 2008: which parties are crucial for decisions on public health measures?» Poster 287. SGIM. Mai 2011. Swiss Medical Forum, suppl. 55, 2011;11(18).

17 Kaelin RM, Diethelm P. Efforts of Industry to influence Tobacco Control Policy in Switzerland. Poster SGIM Mai 2010.

18 Diese Allianz, deren Federführung durch den Direktor des Schweiz. Gewerbeverbandes Hans-Ulrich Bigler, wahrgenommen wird, besteht aus folgenden Mitgliedern: Schweizer. Gewerbeverband, Economiesuisse, ASCO (Verband Schweiz. Konzertlokale, Cabarets, Dancings und Diskotheken), Gastrosuisse, Hotelleriesuisse, Schweizer Bauernverband, Schweizer Casinoverband, SchweizerWerbung, Vereinigung Schweizer Tabakwarenhandel, Verband Schweizer Zigarrenfabrikanten, VISCOM (Schweizerischer Verband für visuelle Kommunikation), u.a. Unter den unterstützenden Organisationen finden sich die Christliche Volkspartei, die Schweizerische Volkspartei, und Swiss Cigarette (welche Philip Morris Internat., British American Tobacco Internat. und Japan Tobacco international umfasst).

19 Dass Wirtevereinigungen gegen Rauchverbote kämpfen, ist eine Konstante der Passivrauchdebatte seit den 90er Jahren in Kalifornien (vergl. Fogel's Testimony, vor dem Stadtrat von New York.: «Es existierte keine Restaurantbesitzervereinigung vor der Rauchfreiheitverordnung; wir wurden durch die Tabakindustrie organisiert.») Die Einflussnahme auf Gastrosuisse und die in der Schweiz domizilierte HoReCa - international lässt sich aus Dokumenten der Tabakindustrie von 1994 und 1995 belegen. Siehe Anmerkungen online $\mathrm{zu}$ «Passivrauchgesetze: Die Bayern haben sich gegen die spanische Konfusion entschieden. Schweiz Ärztezeitung. 2010; 91(41): 1618-20. 